\title{
Model Organisms Reveal Insight into Human Neurodegenerative Disease: Ataxin-2 Intermediate-Length Polyglutamine Expansions Are a Risk Factor for ALS
}

\author{
Nancy M. Bonini • Aaron D. Gitler
}

Received: 29 March 2011 /Accepted: 6 May 2011 / Published online: 10 June 2011

(C) The Author(s) 2011. This article is published with open access at Springerlink.com

\begin{abstract}
Model organisms include yeast Saccromyces cerevisae and fly Drosophila melanogaster. These systems have powerful genetic approaches, as well as highly conserved pathways, both for normal function and disease. Here, we review and highlight how we applied these systems to provide mechanistic insight into the toxicity of TDP-43. TDP-43 accumulates in pathological aggregates in ALS and about half of FTD. Yeast and fly studies revealed an interaction with the counterparts of human Ataxin-2, a gene whose polyglutamine repeat expansion is associated with spinocerebellar ataxia type 2 . This finding raised the hypothesis that repeat expansions in ataxin-2 may associate with diseases characterized by TDP-43 pathology such as ALS. DNA analysis of patients revealed that intermediatelength polyglutamine expansions in ataxin-2 are a risk factor for ALS, such that repeat lengths are greater than normal, but lower than that associated with spinocerebellar ataxia type 2 (SCA2), and are more frequent in ALS patients than in matched controls. Moreover, repeat expansions associated with ALS are interrupted CAACAG sequences as opposed to the pure CAG repeat expansions typically associated with SCA2. These studies provide an example of how model systems, when extended to human cells and human patient tissue, can reveal new mechanistic insight into disease.
\end{abstract}

\footnotetext{
N. M. Bonini $(\bowtie)$

Department of Biology,

University of Pennsylvania,

Philadelphia, PA 19104, USA

e-mail: nbonini@sas.upenn.edu

\section{A. D. Gitler}

Department of Cell and Developmental Biology,

University of Pennsylvania School of Medicine,

Philadelphia, PA 19104, USA

e-mail: gitler@mail.med.upenn.edu
}

Keywords Drosophila $\cdot$ Yeast · ALS · Ataxin-2 .

Polyglutamine disease $\cdot$ Repeat expansions $\cdot$ TDP-43

\section{Introduction}

Human Neurodegenerative Disease and its Challenges

Human neurodegenerative diseases include disorders such as Alzheimer's disease, Parkinson's disease, amyotrophic lateral sclerosis (ALS), and frontotemporal lobular dementia (FTLD; for recent reviews, see Lagier-Tourenne and Cleveland 2009; Chen-Plotkin et al. 2010; Dawson et al. 2010; Reitz et al. 2011). Although these diseases are typically sporadic in nature, familial forms exist that allow the identification of key gene products that may be involved in both familial as well as sporadic situations. In addition, it is often the case that the protein products of genes in which mutations can be associated with disease are also found to accumulate abnormally in at least some percentage of the disease situations. For example, rare mutations in alpha-synuclein are associated with familial Parkinson's disease, and sporadic Parkinson's disease is typified by Lewy bodies comprised of alpha-synuclein. The abnormal accumulation of Tar DNA-binding protein of $43 \mathrm{kD}$ (TDP-43) typifies most ALS and about half of FTLD (FTLD-TDP), and mutations in TDP-43 occur in ALS and in rare cases of FTLD (Lagier-Tourenne and Cleveland 2009; Chen-Plotkin et al. 2010). Further, these studies highlight the notion that the abnormal accumulation, and/or localization of such gene products, associates with disease and may be causal. These types of findings pave the way for modeling such disorders in other simpler systems. Whereas such systems include human or mammalian cells in culture and the mouse, more fundamental model 
organisms such as yeast and Drosophila have extremely powerful techniques that can be brought to bear on these problems. Here, we focus on our recent insights revealed by yeast and fly in the study of TDP-43 toxicity.

\section{Model Organisms Bring Insight into Human Disease}

Model organisms include systems such as the simple yeast, fly, and nematode. These systems have highly manipulable and very powerful genetic approaches, which allow insight to be elucidated, and in relatively rapid form, into fundamental gene pathways and protein functions. Classically, these systems have been used to approach key problems, such as cell cycle or secretion (for example, yeast; Nurse 2002; Luzio et al. 2007), and gene function and impact in development (fly and nematode; Putcha and Johnson 2004; Bier 2005). However, their strengths extend well beyond these basic "normal" processes and into disease. With the elucidation of the genomes of these organisms and network interactions, it has become clear that many genes in many different processes are highly conserved with humans (Adams et al. 2000; Boone et al. 2007). This means that these simpler systems can be employed to provide insight into those features that are shared in a process between these systems and the more complex and more difficult to study human situation. Moreover, the impact of model organisms is not limited to normal aspects of development, but one can "re-create" certain aspects of a human disease in these simple systems (Jackson et al. 1998; Warrick et al. 1998; Feany and Bender 2000; Cooper et al. 2006; Johnson et al. 2008). This then allows use of the simpler systems as a launching ground to define key pathways and processes of relevance to the far more complex disease in humans. The key points into generating models for human disease include that many human neurodegenerative diseases are thought to be associated with gain-of-function activities due to the accumulation of the protein of interest. This means that by expressing the wild-type or disease-associated mutant version of the protein linked to the human disease, one hopes to recapitulate aspects of the toxicity of the protein. Although the disease in humans may be complex, and may comprise both gain-of-function as well as loss-of-function aspects of the disease protein, it is possible to get a handle on features of the toxicity of the disease protein.

The strength of simple model organisms for providing "genome-wide" insight into the disease process cannot be overstated. For example, the yeast genetics armamentarium is bafflingly powerful: entire libraries exist of every gene of the genome knocked out or upregulated. Thus, once a model is in hand, one can assess the effects of changing gene dosage of every gene of the genome. For Drosophila, the large collections of upregulation or reduced gene activity lines exist, which can similarly be used to assess for activity on the disease effect in the fly. Whereas, yeast has the advantage of a single cell, allowing a simple view of processes and pathways, Drosophila offers the added complexity and interactions of neurons, and integrity of the nervous system and brain with age.

\section{Yeast as a Model for Human Disease: Special Insights into TDP-43 Toxicity}

To provide a foundation for yeast to reveal insight into TDP-43 toxicity, the human gene, tagged for ready visualization with fluorescent YFP, was expressed in yeast using a conditional system (Johnson et al. 2008). The expression of TDP-43 at low levels causes the protein to be nuclear, with occasional foci formation; markedly, expression at higher levels dramatically shifts the localization to the cytoplasm with notable foci. Intriguingly, in ALS and FTLD-TDP, TDP-43, which is normally nuclear, is now seen also in the cytoplasm forming round or skein-like inclusions (Neumann et al. 2006). Thus, aggregation of the protein can be modeled in yeast.

Although accumulation of TDP-43 is noteworthy in disease, the consequences were unclear. But strikingly in yeast, TDP-43 is toxic, leading to loss of growth of the cells (Johnson et al. 2008). This fundamental finding thus provides the foundation for many additional derivative experiments to explore and define features of the toxicity. For example, what domains of the TDP-43 protein are necessary and sufficient for toxicity? Intriguingly, these studies identify the predicted RNA-binding RRM2 domain and the prion-like $\mathrm{C}$-terminal glycine-rich tail as central (Johnson et al. 2008; Cushman et al. 2010). Notably, most human mutations associated with disease are localized to the C-terminal domain (Lagier-Tourenne and Cleveland 2009). These studies also dissociate aggregation of the protein from toxic interactions, by demonstrating that aggregation into foci is not sufficient for toxicity in yeast.

More detailed study reveals that C-terminal mutations associated with ALS accelerate the aggregation of the protein in yeast, such that most mutations present with a greater number of foci at the same time point, compared to the wildtype version of the protein (Johnson et al. 2009). Compelling additional data raise the hypothesis that proteins like TDP43, and another protein associated with ALS, FUS, have domains that are prion-like in nature, being rich in nonpolar amino acids, asparagine, glutamine, and tyrosine (Cushman et al. 2010). Perhaps this is a common feature of proteins associated with human neurodegenerative disease, because of their propensity to form aggregates.

Notably, different human neurodegenerative disease proteins, when expressed in yeast, can cause toxicity. For 
example, both alpha-synuclein (Cooper et al. 2006) and TDP-43 (Johnson et al. 2008) cause a failure of growth. Superficially, these proteins act in a similar way, in that both proteins aggregate and are toxic in yeast. However, the network of interactions underscores that the nature of the toxicity is distinct: a comparison of genome-wide modifiers of alpha-synuclein toxicity and TDP-43 toxicity show little overlap (Cooper et al. 2006; Gitler et al. 2009; Yeger-Lotem et al. 2009; Elden et al. 2010). As with any such network, the challenge then becomes determining what the network of interacting proteins is revealing, and which one or ones are worthy of aggressive focus.

\section{Drosophila as a Model for Human Disease: Special Insights into Disease Processes}

Drosophila is a complex organism, complete with a functioning nervous system and brain, capable of many behaviors like learning, motility, and visual acuity. This system has become popular for the study of human neurodegenerative disease proteins (Shulman et al. 2003; Bilen and Bonini 2005; Marsh and Thompson 2006). Select techniques or types of analyses can be emphasized to highlight those features that reflect the human disease situation, thus tailoring the approach. Typically, to achieve expression mimicking a gain-of-function human neurodegenerative disease situation, the protein of interest is upregulated in the fly. One of the standard systems used is the GAL4-UAS approach, where the gene of interest is selectively driven in select tissues using GAL4 drivers (drivers that direct expression to the eye, or nervous system, or even a particular class of neuron; Brand and Perrimon 1993). This is a gain-of-function approach that can also be combined with loss of gene function approaches in order to explore whether the toxicity of the protein is pure gain of function or is coupled with reduced activity, as well. These types of experiments can also address whether the human protein is able to confer function in the Drosophila system by replacing the Drosophila gene. Behaviors that are typically assessed include lifespan of the animal: does the protein cause reduced survival with age? Motility of the animal: does the protein cause more rapid loss of climbing function with age? One can also compare toxicity of different versions of the same protein expressed at the same level: are mutations in TDP-43 more toxic than the wild-type protein? Does the protein accumulate abnormally, as in the human disease, and in the cytoplasm or nucleus? Does manipulating its localization matter? Finally, a common tissue to assess is the eye of the fly, simply for the genetic applications. The eye has the advantage of being nonessential to the organism, but highly manipulable and readily scored in screens for greater or lessened toxicity, if the protein has an effect on the external structure. Thus, by combining these various techniques and approaches, and comparing the range and the type of effects with the human disease, one can establish a model that reflects fundamental features of the disease.

A number of models for TDP-43 toxicity in flies have been generated (Elden et al. 2010; Li et al. 2010; Ritson et al. 2010; Voigt et al. 2010), revealing that the protein shows toxicity in vivo. Indeed, the upregulation of wild-type TDP43 , or the Q331K mutant, causes progressive degeneration in the eye, coupled with the loss of motility and lifespan when expressed in the nervous system or directed to motor neurons (Elden et al. 2010). Interestingly, the loss of TDP-43 also causes deficits (Feiguin et al. 2009), suggesting that a careful analysis of TDP-43 toxicity should ultimately include both studies to reveal gain-of-function activities, as well as lossof-function effects.

\section{Ataxin-2 is a Modifier of TDP-43 Toxicity}

Combining the power of yeast and fly promises to reveal those pathways of disease that overlap and that are most conserved. Thus, to gain insight into mechanisms of TDP43 pathogenesis, an unbiased genetic approach was used in yeast to identify genes that could suppress or enhance TDP43 toxicity (Elden et al. 2010). The largest functional class enriched in the screen included RNA-binding proteins and proteins involved in RNA metabolism, and revealed minimal overlap with alpha-synuclein modifiers. Thus, the modifiers address the selective biology of the disease proteins (e.g., vesicle trafficking for alpha-synuclein and RNA biology with TDP-43), informing insight into pathology in disease.

One gene identified that enhanced TDP-43 toxicity, $P B P 1$ (poly(A)-binding protein, (Pablp)-binding protein), was of immediate interest, as it is the yeast homologue of human ataxin-2, a polyglutamine protein mutated in the human neurodegenerative disease spinocerebellar ataxia type 2 (SCA2; Fig. 1a). Although SCA2 primarily presents with cerebellar Purkinje neuron degeneration, as in ALS, motor neurons can also degenerate, although these features are typically later than the cerebellar degeneration. In select cases, however, the motor neuron features can be sufficiently prominent to mimic an ALS presentation (Infante et al. 2004; Nanetti et al. 2009). These findings raise the potential for mechanistic overlap between SCA2 and ALS. Ataxin-2, like yeast $\mathrm{Pbp} 1$, is likely involved in aspects of RNA metabolism (Mangus et al. 1998; Lastres-Becker et al. 2008). Notably, $\mathrm{Pbp} 1$ is a dose-sensitive modifier of TDP-43 toxicity in yeast, with upregulation enhancing and loss of function suppressing TDP-43 toxicity. These data indicate that the genes are critical interacting components. 
Fig. 1 TDP-43 is toxic to yeast and fly, and ataxin-2 enhances this toxicity. a $\mathrm{Pbp} 1$, the yeast homologue of ataxin-2, is a dosesensitive modifier of TDP-43 toxicity in yeast. Fivefold serial dilutions of yeast cells spotted onto glucose (expression repressed) or galactose (expression induced). Spotting assays with yeast expressing TDP-43 showing toxicity. Upregulation of PBPI enhances TDP-43 toxicity. b Ataxin-2 is a modifier of TDP43 toxicity in the nervous system of Drosophila. Flies expressing TDP-43 or dAtx2 alone (dAtx $\left.2^{E P}\right)$ in the eye (with gmr-GAL4 driver) have a mild effect on retinal structure. TDP-43 toxicity is more severe upon upregulation of dAtx2 $\left(d A t x 2^{E P}\right)$. TDP-43 toxicity is markedly mitigated upon the reduction of dAtx2 (flies in trans to null allele $d A t x 2^{X I}$ ). The figure and the legend are from Elden et al. (2010) a

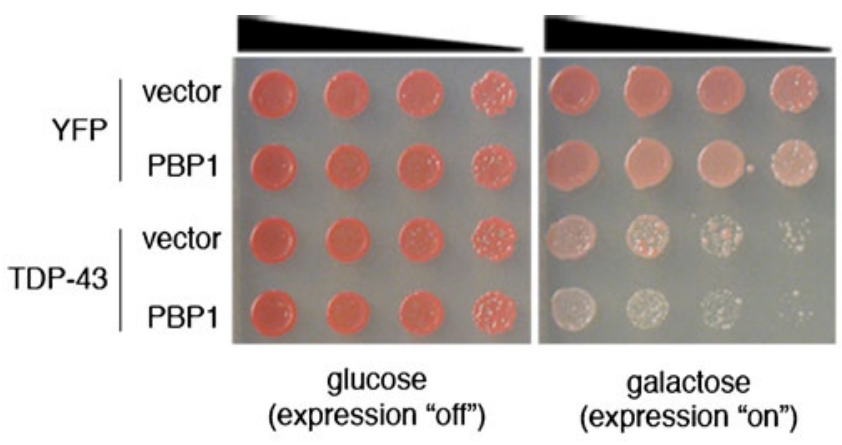

b
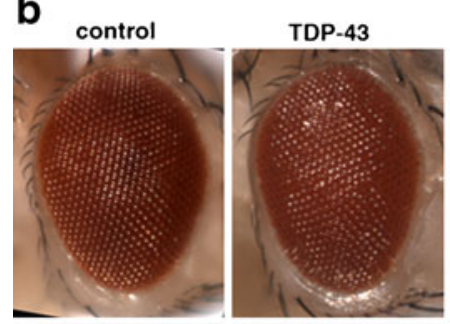

$d A t \times 2^{E P}$

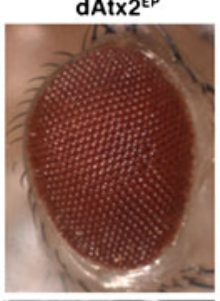

TDP $-43+$
dAt $\times 2^{E P}$

TDP-43;

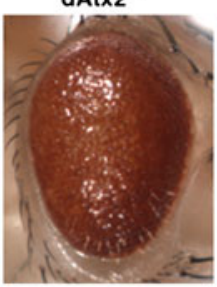
dAt $\times 2^{x_{1}} /+$

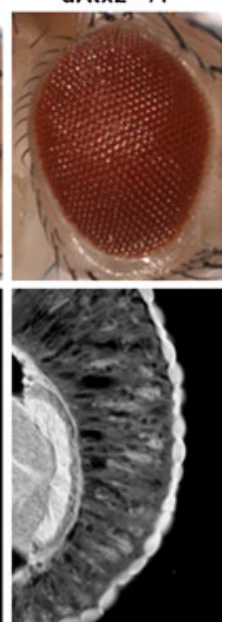

Drosophila can reveal the relevance to the intact, functioning nervous system (Fig. 1b). Thus potential interactions between TDP-43 and ataxin-2 were extended to the fly. Moreover, the fly also has an ataxin-2 homologue, thus one can assess dose-sensitive interactions with the endogenous gene. As noted above, TDP-43 causes retinal degeneration, loss of motility, and reduced lifespan. Remarkably, testing for interactions with the fly ataxin-2 revealed that upregulation of the fly gene dramatically potentiated toxicity of TDP-43, leading to more severe retinal degeneration, further shortening of lifespan, and causing more rapid loss of motility (Fig. 1b; Elden et al. 2010). Strikingly, reduction of ataxin-2 also had a dramatic effect, in this case to mitigate toxicity, extending lifespan to nearly normal levels. Thus, these findings indicate that the modulation of TDP-43 toxicity by ataxin- 2 extends to the nervous system, where it is exquisitely sensitive to the levels of ataxin-2.

These studies were extended to assess the interaction between the two proteins in cells in culture and patient tissue (Elden et al. 2010). In HEK293 T cells, TDP-43 is normally nuclear, but targeting the protein to the cytoplasm leads to foci formation. These foci co-localize with ataxin-2. Further, upregulated TDP-43 interacts with endogenous ataxin-2 protein by co-immunoprecipitation. This interaction, by coimmunoprecipitation or immunocytochemistry, is dependent upon the RNA-binding capacity of the protein, as revealed by point mutation of the RRM domains in the TDP-43 protein. Further, the toxicity in yeast is similarly dependent upon the RNA-binding capacity of the protein. Thus, these findings indicate the proteins interact biologically by genetic and biochemical approaches, and that the RNA-binding activities of TDP-43 are important for these interactions.

Analysis of postmortem spinal cord sections of ALS patients and controls further support an interaction. First, ataxin-2 is robustly expressed in spinal cord motor neurons, as is TDP-43. Second, although ataxin- 2 is normally localized in a diffuse or fine granular pattern, in $\sim 30 \%$ of motor neurons of ALS patients, ataxin-2 shows larger, more distinct accumulations (Fig. 2; Elden et al. 2010). Finally, ataxin-2 can be seen to overlap with TDP-43 immunostaining in cortical inclusions in FTLD-TDP, although not in the spinal cord in ALS.

\section{Ataxin-2 Intermediate-Length PolyQ Expansions Are a Risk Factor for ALS}

Our initial interest in ataxin- 2 was spurred by an understanding of the mutations in ataxin- 2 that are associated with human disease. That is, ataxin-2 bears a polyglutamine repeat that, upon expansion, causes SCA2 disease. However, there is a 

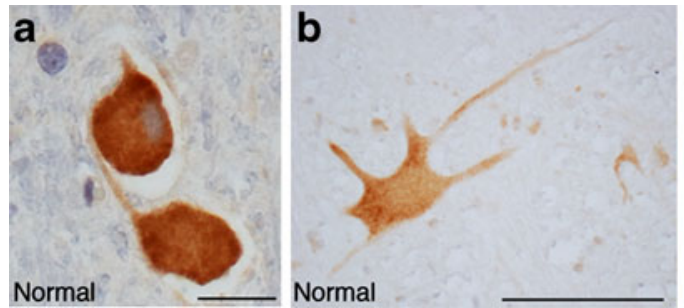

Fig. 2 Ataxin-2 shows altered localization in spinal cord motor neurons of ALS patients. Immunostaining for ataxin-2 in spinal cord. a, b In control spinal cord neurons, ataxin-2 is localized throughout the cytoplasm in a diffuse pattern. c, d In ALS spinal cord neurons,

gap between the polyglutamine length of normal and SCA2. The polyglutamine tract length in ataxin- 2 is most frequently 22-23, with expansions of greater than 34 being associated with SCA2 (Imbert et al. 1996; Pulst et al. 1996; Sanpei et al. 1996; Lorenzetti et al. 1997). Thus, could it be that polyQ repeat lengths that are greater than normal, but below the threshold for SCA2, are associated with ALS?

Genescan analysis was used to define ataxin-2 polyglutamine repeat lengths in genomic DNA from more than 900 individuals diagnosed with ALS and a similar number of ethnically and geographically matched neurologically normal controls. This analysis revealed an association between longer repeat lengths (>26-33) in ataxin-2 and ALS (Fig. 3). Detailed analysis determined that whereas only $1.4 \%$ of controls bear a single intermediate-length ataxin-2 allele, $4.7 \%$ of ALS patients had one allele with an intermediatelength ataxin-2 repeat. These included both sporadic and

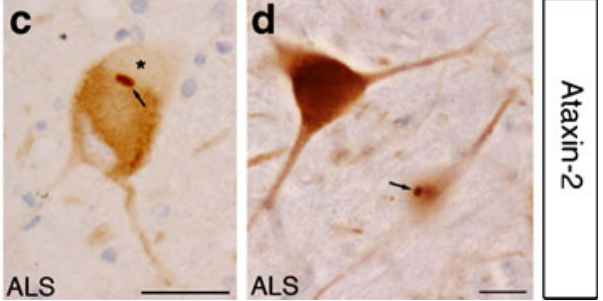

ataxin-2 was present in distinct cytoplasmic accumulations (arrows). In some cases, ataxin-2 positive accumulations were adjacent to clearings indicative of TDP-43 aggregates (asterisk in b). The figure and the legend are from Elden et al. (2010)

familial disease cases. Notably, in 980 neurologically normal controls, only three individuals had expansions $>28$ (all three were under the age of 60), whereas 24 ALS cases fell in that range. These studies therefore indicated that intermediatelength ataxin-2 polyglutamine repeat lengths are significantly associated with risk for ALS.

The extension of these findings to other populations indicates that an association between longer polyglutamine repeats in ataxin-2 and ALS also occurs, although the frequency and precise repeat length cutoff appears dependent on the specific population. Thus, in Europeans, there is also an increased risk for ALS with longer polyglutamine repeats in ataxin-2; however, the repeat length cutoff is greater than 30 glutamines, such than $1 \%$ of the patients harbor such repeats (Lee et al. 2011b).

Detailed studies reveal insight into the interaction between the two proteins, as well as complexities that may underlie
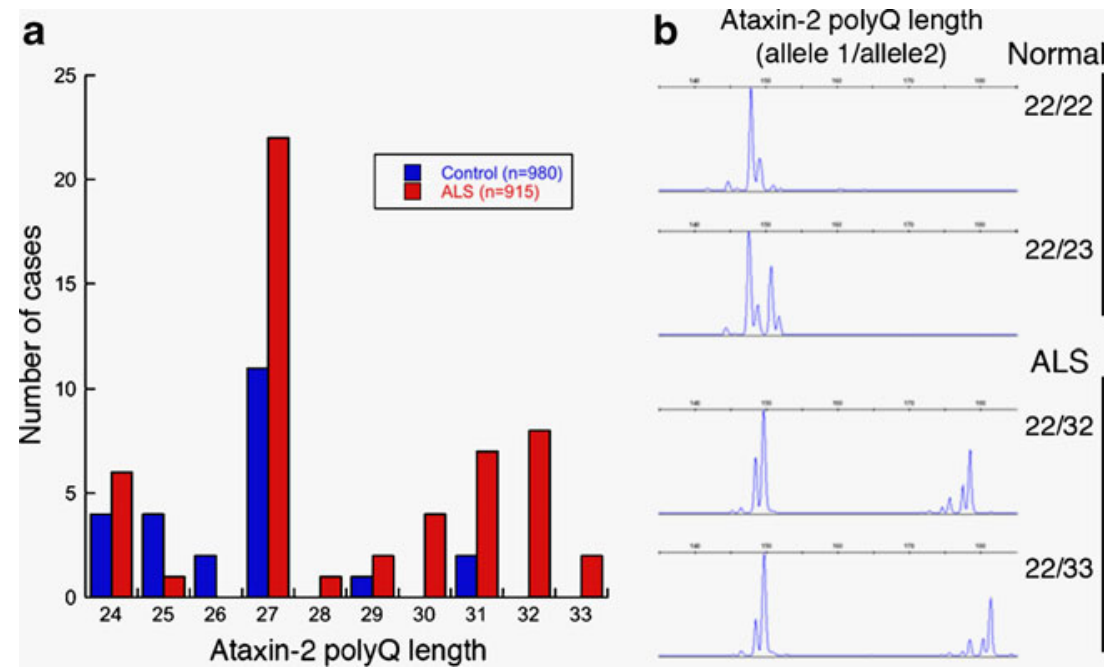

Fig. 3 Ataxin-2 intermediate-length polyglutamine repeat expansions are associated with increased risk for ALS. The ATXN2 gene contains a trinucleotide repeat encoding polyglutamine. The repeat length is normally 22-23Q. Expansions of $>34$ cause SCA2. We hypothesized that the intermediate-length polyglutamine expansions could be linked to ALS. The ataxin-2 polyglutamine length was defined by Genescan analysis of ALS cases and neurologically normal controls. a The distribution of ataxin-2 polyglutamine repeat lengths in ALS and control cases. Polyglutamine lengths $\geq 27$ are significantly enriched in ALS. Further, polyQ lengths $>31$ were never observed in our controls, but we found ten ALS patients above this threshold. b Representative examples of Genescan analysis of polyglutamine lengths from control and ALS cases. The figure and the legend are from Elden et al. (2010) 
disease. Both TDP-43 and ataxin-2 can localize to stress granules and interact with stress granule components (Nonhoff et al. 2007; Liu-Yesucevitz et al. 2010). Thus, it stands to reason that the proteins may interact in stress granules and notably, that one catalyst for interaction may be stress. Strikingly, heat shock induces some TDP-43 to accumulate in the cytoplasm of cells normally; in beta-lymphoblasts derived from controls versus ALS patients with normal and expanded ataxin-2 repeat lengths, stress induces a greater percentage of cells to accumulate TDP-43 in the cytoplasm (Elden et al. 2010). The fly and yeast gene dosage data suggest that ataxin- 2 and TDP-43 normally interact, and together with cellular data, these interactions may become more severe upon ataxin-2 repeat expansions and stress.

Ataxin-2 also presents a fascinating human disease gene because it can present with multiple clinical presentations. Expansions greater than 34 are associated with SCA2, and we have suggested that repeats that are greater than normal, but less than that associated with SCA2, are a genetic risk factor for ALS. However, expansions in ataxin-2 can also present atypically with parkinsonism (Giunti et al. 1998; GwinnHardy et al. 2000; Shan et al. 2001; Furtado et al. 2002; Lu et al. 2004; Ragothaman et al. 2004; Boone et al. 2007; Modoni et al. 2007; Socal et al. 2009). Intriguingly, it has been noted in at least some of these situations that the repeat expansions in the gene encoding ataxin-2 may be interrupted CAG expansions versus the pure CAG repeat expansions of SCA2. That is, expansions in ataxin-2 associated with SCA2 are typically pure CAG repeat expansions, whereas those associated with parkinsonism have been noted to be CAA or other codon interruptions (Gwinn-Hardy et al. 2000). Since CAG and CAA codons both specify the amino acid glutamine, it suggests the intriguing possibility that the particular configuration of the RNA encoding the polyglutamine tract can also play a role in pathogenesis. This raises the question whether the repeat expansions in ataxin-2 associated with ALS are pure CAG repeats or interrupted repeats. Notably, pure CAG repeat expansions can confer toxicity on their own ( $\mathrm{Li}$ et al. 2008); the mechanisms are of great interest and may include interactions with select proteins and noninitiation-dependent translation ( $\mathrm{Li}$ and Bonini 2010; $\mathrm{Zu}$ et al. 2011). Analysis of the repeat sequences in alleles associated with increased risk for ALS reveals that the repeats are interrupted, thus not pure CAG repeats (Yu et al. 2011). Thus, the integration of RNAbased toxicity due to the RNA encoding the disease protein, as well as protein-based effects, may be involved.

\section{Conclusions/Future Perspectives}

The application of model organism strengths to the problems of human disease have allowed insight into numerous pathways and processes that go awry in disease, as well as allowed focus for disease therapeutics. Of course, not all aspects of the disease will be recapitulated, as these systems have fewer genes and less complexity including in the nervous system. However, the use of these systems will allow focus on those pathways that are conserved, allowing a key insight into the fundamental processes affected. These studies to reveal insight into TDP-43 using yeast and fly have highlighted an important new player in ALS in ataxin-2. Does ataxin-2 also play a role in FTD, where TDP-43 is also prominent? Does ataxin-2 also play a role in other human diseases, such as Parkinson's and Alzheimer's diseases, which can have clinical overlaps and also show protein pathological overlaps? What about other polyglutamine disease genes in ALS? Our studies have revealed a lack of association of expanded polyglutamine repeat lengths in other polyglutamine genes in ALS, indicating specificity to ataxin-2 (Lee et al. 2011a). However, it remains possible that normal activities of other polyglutamine disease proteins intersect in ALS. The integration of model organism studies with human pathological insight will reveal key gene nodes that underlie many of these diseases, defining central players to attack therapeutically. Future studies are focused on detailed assessment of the interactions between TDP-43, ataxin-2, and the combined toxicity, as well as more insight into other pathways and processes affected. With such approaches, and the combined insight of genome wide association studies and next generation sequencing approaches, we anticipate revealing all the genes that can contribute to these diseases soon, with the following challenge to determine how the pathways intertwine and overlap, and go awry in disease.

Acknowledgments This work is supported by the University of Pennsylvania Institute on Aging (A.D.G.), an NIH Director's New Innovator Award 1DP2OD004417-01 (A.D.G.), the NIH 1R01NS06531701 (A.D.G.), the NINDS R01-NS043578 (N.M.B.), and an Ellison Medical Foundation Senior Scholar Award (N.M.B.). A.D.G. is a Pew Scholar in the Biomedical Sciences supported by The Pew Charitable Trusts. N.M.B. is an Investigator of the Howard Hughes Medical Institute. We are truly grateful for the dedication of the patients and their families and for their invaluable contributions to our research.

Open Access This article is distributed under the terms of the Creative Commons Attribution Noncommercial License which permits any noncommercial use, distribution, and reproduction in any medium, provided the original author(s) and source are credited.

\section{References}

Adams MD et al (2000) The genome sequence of Drosophila melanogaster. Science 287:2185-2195

Bier E (2005) Drosophila, the golden bug, emerges as a tool for human genetics. Nat Rev Genet 6:9-23

Bilen J, Bonini NM (2005) Drosophila as a model for human neurodegenerative disease. Annu Rev Genet 39:153-171 
Boone C, Bussey H, Andrews BJ (2007) Exploring genetic interactions and networks with yeast. Nat Rev Genet 8:437-449

Brand AH, Perrimon N (1993) Targeted gene expression as a means of altering cell fates and generating dominant phenotypes. Development 118:401-415

Chen-Plotkin AS, Lee VM, Trojanowski JQ (2010) TAR DNAbinding protein 43 in neurodegenerative disease. Nat Rev Neurol 6:211-220

Cooper AA, Gitler AD, Cashikar A, Haynes CM, Hill KJ, Bhullar B, Liu K, Xu K, Strathearn KE, Liu F, Cao S, Caldwell KA, Caldwell GA, Marsischky G, Kolodner RD, Labaer J, Rochet JC, Bonini NM, Lindquist S (2006) Alpha-synuclein blocks ER-Golgi traffic and Rab1 rescues neuron loss in Parkinson's models. Science 313:324328

Cushman M, Johnson BS, King OD, Gitler AD, Shorter J (2010) Prionlike disorders: blurring the divide between transmissibility and infectivity. J Cell Sci 123:1191-1201

Dawson TM, Ko HS, Dawson VL (2010) Genetic animal models of Parkinson's disease. Neuron 66:646-661

Elden AC et al (2010) Ataxin-2 intermediate-length polyglutamine expansions are associated with increased risk for ALS. Nature 466:1069-1075

Feany MB, Bender WW (2000) A Drosophila model of Parkinson's disease. Nature 404:394-398

Feiguin F, Godena VK, Romano G, D'Ambrogio A, Klima R, Baralle FE (2009) Depletion of TDP-43 affects Drosophila motoneurons terminal synapsis and locomotive behavior. FEBS Lett 583:1586-1592

Furtado S, Farrer M, Tsuboi Y, Klimek ML, de la Fuente-Fernandez R, Hussey J, Lockhart P, Calne DB, Suchowersky O, Stoessl AJ, Wszolek ZK (2002) SCA-2 presenting as parkinsonism in an Alberta family: clinical, genetic, and PET findings. Neurology 59:1625-1627

Gitler AD, Chesi A, Geddie ML, Strathearn KE, Hamamichi S, Hill KJ, Caldwell KA, Caldwell GA, Cooper AA, Rochet JC, Lindquist S (2009) Alpha-synuclein is part of a diverse and highly conserved interaction network that includes PARK9 and manganese toxicity. Nat Genet 41:308-315

Giunti P, Sabbadini G, Sweeney MG, Davis MB, Veneziano L, Mantuano E, Federico A, Plasmati R, Frontali M, Wood NW (1998) The role of the SCA2 trinucleotide repeat expansion in 89 autosomal dominant cerebellar ataxia families. Frequency, clinical and genetic correlates. Brain 121(Pt 3):459-467

Gwinn-Hardy K, Chen JY, Liu HC, Liu TY, Boss M, Seltzer W, Adam A, Singleton A, Koroshetz W, Waters C, Hardy J, Farrer M (2000) Spinocerebellar ataxia type 2 with parkinsonism in ethnic Chinese. Neurology 55:800-805

Imbert G, Saudou F, Yvert G, Devys D, Trottier Y, Garnier JM, Weber C, Mandel JL, Cancel G, Abbas N, Durr A, Didierjean O, Stevanin G, Agid Y, Brice A (1996) Cloning of the gene for spinocerebellar ataxia 2 reveals a locus with high sensitivity to expanded CAG/glutamine repeats. Nat Genet 14:285-291

Infante J, Berciano J, Volpini V, Corral J, Polo JM, Pascual J, Combarros O (2004) Spinocerebellar ataxia type 2 with Levodopa-responsive parkinsonism culminating in motor neuron disease. Mov Disord 19:848-852

Jackson GR, Salecker I, Dong X, Yao X, Arnheim N, Faber PW, MacDonald ME, Zipursky SL (1998) Polyglutamine-expanded human huntingtin transgenes induce degeneration of Drosophila photoreceptor neurons. Neuron 21:633-642

Johnson BS, McCaffery JM, Lindquist S, Gitler AD (2008) A yeast TDP-43 proteinopathy model: exploring the molecular determinants of TDP-43 aggregation and cellular toxicity. Proc Natl Acad Sci USA 105:6439-6444

Johnson BS, Snead D, Lee JJ, McCaffery JM, Shorter J, Gitler AD (2009) TDP-43 is intrinsically aggregation-prone, and amyotro- phic lateral sclerosis-linked mutations accelerate aggregation and increase toxicity. J Biol Chem 284:20329-20339

Lagier-Tourenne C, Cleveland DW (2009) Rethinking ALS: the FUS about TDP-43. Cell 136:1001-1004

Lastres-Becker I, Rub U, Auburger G (2008) Spinocerebellar ataxia 2 (SCA2). Cerebellum 7:115-124

Lee T, Li YR, Chesi A, Hart MP, Ramos D, Jethava N, Hosangadi D, Epstein J, Hodges B, Bonini NM, Gitler AD (2011a) Evaluating the prevalence of polyglutamine repeat expansions in amyotrophic lateral sclerosis. Neurology (in press).

Lee T, Li YR, Ingre C, Weber M, Grehl T, Gredal O, de Carvalho M, Meyer T, Tysnes OB, Auburger G, Gispert S, Bonini NM, Andersen PM, Gitler AD (2011b) Ataxin-2 intermediate-length polyglutamine expansions in European ALS patients. Hum Mol Genet 20:1697-1700

Li LB, Bonini NM (2010) Roles of trinucleotide-repeat RNA in neurological disease and degeneration. Trends Neurosci 33:292298

Li LB, Yu Z, Teng X, Bonini NM (2008) RNA toxicity is a component of ataxin-3 degeneration in Drosophila. Nature 453:1107-1111

Li Y, Ray P, Rao EJ, Shi C, Guo W, Chen X, Woodruff EA 3rd, Fushimi K, Wu JY (2010) A Drosophila model for TDP-43 proteinopathy. Proc Natl Acad Sci USA 107:3169-3174

Liu-Yesucevitz L, Bilgutay A, Zhang YJ, Vanderwyde T, Citro A, Mehta T, Zaarur N, McKee A, Bowser R, Sherman M, Petrucelli L, Wolozin B (2010) Tar DNA binding protein-43 (TDP-43) associates with stress granules: analysis of cultured cells and pathological brain tissue. PLoS ONE 5:e13250

Lorenzetti D, Bohlega S, Zoghbi HY (1997) The expansion of the CAG repeat in ataxin-2 is a frequent cause of autosomal dominant spinocerebellar ataxia. Neurology 49:1009-1013

Lu CS, Wu Chou YH, Kuo PC, Chang HC, Weng YH (2004) The parkinsonian phenotype of spinocerebellar ataxia type 2. Arch Neurol 61:35-38

Luzio JP, Pryor PR, Bright NA (2007) Lysosomes: fusion and function. Nat Rev Mol Cell Biol 8:622-632

Mangus DA, Amrani N, Jacobson A (1998) Pbplp, a factor interacting with Saccharomyces cerevisiae poly(A)-binding protein, regulates polyadenylation. Mol Cell Biol 18:7383-7396

Marsh JL, Thompson LM (2006) Drosophila in the study of neurodegenerative disease. Neuron 52:169-178

Modoni A, Contarino MF, Bentivoglio AR, Tabolacci E, Santoro M, Calcagni ML, Tonali PA, Neri G, Silvestri G (2007) Prevalence of spinocerebellar ataxia type 2 mutation among Italian Parkinsonian patients. Mov Disord 22:324-327

Nanetti L, Fancellu R, Tomasello C, Gellera C, Pareyson D, Mariotti C (2009) Rare association of motor neuron disease and spinocerebellar ataxia type 2 (SCA2): a new case and review of the literature. J Neurol 256:1926-1928

Neumann M, Sampathu DM, Kwong LK, Truax AC, Micsenyi MC, Chou TT, Bruce J, Schuck T, Grossman M, Clark CM, McCluskey LF, Miller BL, Masliah E, Mackenzie IR, Feldman H, Feiden W, Kretzschmar HA, Trojanowski JQ, Lee VM (2006) Ubiquitinated TDP-43 in frontotemporal lobar degeneration and amyotrophic lateral sclerosis. Science 314:130-133

Nonhoff U, Ralser M, Welzel F, Piccini I, Balzereit D, Yaspo ML, Lehrach H, Krobitsch S (2007) Ataxin-2 interacts with the DEAD/H-box RNA helicase DDX6 and interferes with P-bodies and stress granules. Mol Biol Cell 18:1385-1396

Nurse P (2002) Cyclin dependent kinases and cell cycle control (nobel lecture). Chembiochem 3:596-603

Pulst SM, Nechiporuk A, Nechiporuk T, Gispert S, Chen XN, LopesCendes I, Pearlman S, Starkman S, Orozco-Diaz G, Lunkes A, DeJong P, Rouleau GA, Auburger G, Korenberg JR, Figueroa C, Sahba S (1996) Moderate expansion of a normally biallelic 
trinucleotide repeat in spinocerebellar ataxia type 2. Nat Genet 14:269-276

Putcha GV, Johnson EM Jr (2004) Men are but worms: neuronal cell death in C elegans and vertebrates. Cell Death Differ 11:38-48

Ragothaman M, Sarangmath N, Chaudhary S, Khare V, Mittal U, Sharma S, Komatireddy S, Chakrabarti S, Mukerji M, Juyal RC, Thelma BK, Muthane UB (2004) Complex phenotypes in an Indian family with homozygous SCA2 mutations. Ann Neurol 55:130-133

Reitz C, Brayne C, Mayeux R (2011) Epidemiology of Alzheimer disease. Nat Rev Neurol 7:137-152

Ritson GP, Custer SK, Freibaum BD, Guinto JB, Geffel D, Moore J, Tang W, Winton MJ, Neumann M, Trojanowski JQ, Lee VM, Forman MS, Taylor JP (2010) TDP-43 mediates degeneration in a novel Drosophila model of disease caused by mutations in VCP/p97. J Neurosci 30:7729-7739

Sanpei K et al (1996) Identification of the spinocerebellar ataxia type 2 gene using a direct identification of repeat expansion and cloning technique, DIRECT. Nat Genet 14:277-284

Shan DE, Soong BW, Sun CM, Lee SJ, Liao KK, Liu RS (2001) Spinocerebellar ataxia type 2 presenting as familial levodoparesponsive parkinsonism. Ann Neurol 50:812-815

Shulman JM, Shulman LM, Weiner WJ, Feany MB (2003) From fruit fly to bedside: translating lessons from Drosophila models of neurodegenerative disease. Curr Opin Neurol 16:443-449
Socal MP, Emmel VE, Rieder CR, Hilbig A, Saraiva-Pereira ML, Jardim LB (2009) Intrafamilial variability of Parkinson phenotype in SCAs: novel cases due to SCA2 and SCA3 expansions. Parkinsonism Relat Disord 15:374-378

Voigt A, Herholz D, Fiesel FC, Kaur K, Muller D, Karsten P, Weber SS, Kahle PJ, Marquardt T, Schulz JB (2010) TDP-43-mediated neuron loss in vivo requires RNA-binding activity. PLoS ONE 5: e12247

Warrick JM, Paulson HL, Gray-Board GL, Bui QT, Fischbeck KH, Pittman RN, Bonini NM (1998) Expanded polyglutamine protein forms nuclear inclusions and causes neural degeneration in Drosophila. Cell 93:939-949

Yeger-Lotem E, Riva L, Su LJ, Gitler AD, Cashikar AG, King OD, Auluck PK, Geddie ML, Valastyan JS, Karger DR, Lindquist S, Fraenkel E (2009) Bridging high-throughput genetic and transcriptional data reveals cellular responses to alpha-synuclein toxicity. Nat Genet 41:316-323

Yu Z, Zhu Y, Chen-Plotkin AS, Clay-Falcone D, McCluskey L, Elman L, Kalb RG, Trojanowski JQ, Lee VMY, Van Deerlin VM, Gitler AD, Bonini NM (2011) PolyQ repeat expansions in ATXN2 associated with ALS are CAA interrupted repeats. PLoS ONE 6: e17951

$\mathrm{Zu} \mathrm{T}$ et al (2011) Non-ATG-initiated translation directed by microsatellite expansions. Proc Natl Acad Sci USA 108:260-265 